\title{
A novel role for STOMATAL CARPENTER 1 in stomata patterning
}

\author{
Giulia Castorina ${ }^{1}$, Samantha Fox ${ }^{2}$, Chiara Tonelli ${ }^{1}$, Massimo Galbiati ${ }^{*}$ and Lucio Conti ${ }^{*}$ (D)
}

\begin{abstract}
Background: Guard cells (GCs) are specialised cells within the plant epidermis which form stomatal pores, through which gas exchange can occur. The GCs derive through a specialised lineage of cell divisions which is specified by the transcription factor SPEECHLESS (SPCH), the expression of which can be detected in undifferentiated epidermal cells prior to asymmetric division. Other transcription factors may act before GC specification and be required for correct GC patterning. Previously, the DOF transcription factor STOMATAL CARPENTER 1 (SCAP1) was shown to be involved in GC function, by activating a set of GC-specific genes required for GC maturation and activity. It is thus far unknown whether SCAP1 can also affect stomatal development.

Results: Here we show that SCAP1 expression can also be observed in young leaf primordia, before any GC differentiation occurs. The study of transgenic plants carrying a proSCAP1:GUS-GFP transcriptional fusion, coupled with GPCR analyses, indicate that SCAP1 expression peaks in a temporal window which is coincident with expression of stomatal patterning genes. Independent scap1 loss-of-function mutants have a reduced number of GCs whilst SCAP1 over expression lines have an increased number of GCS, in addition to altered GC distribution and spacing patterns. The study of early markers for stomatal cell lineage in a background carrying gain-of-function alleles of SCAP1 revealed that, compared to the wild type, an increased number of protodermal cells are recruited in the GC lineage, which is reflected in an increased number of meristemoids.
\end{abstract}

Conclusions: Our results suggest an early role for SCAP1 in GC differentiation. We propose that a function of SCAP1 is to integrate different aspects of GC biology including specification, spacing, maturation and function.

Keywords: Arabidopsis, SCAP1 (AT5G65590), Guard Cells development, DOF-type transcription factors, SPCH (AT5G53210), AtMYB60 (AT1G08810)

\section{Background}

Guard cells (GCs) are specialised epidermal cells which form stomatal pores, through which gas exchange can occur. Since transpiration is linked to plant growth and survival, control of GC number, distribution and activity is tightly regulated. Mature GC pairs form in the epidermal cell layer and originate from a single undifferentiated protodermal cell (PDC). Each PDC undergoes a series of cell divisions and successive cell-state transitions. These transitional states are characterized by changes in cell morphology and are associated with alterations in the transcriptomic signature [1-3]. A subset of PDCs, termed meristemoid mother cells - MMCs -, become

\footnotetext{
*Correspondence: massimo.galbiati@unimi.it; lucio.conti@unimi.it ${ }^{1}$ Dipartimento di Bioscienze, Università degli studi di Milano, Via Celoria 26, 20133 Milan, Italy

Full list of author information is available at the end of the article
}

competent to initiate the stomatal cell lineage. The MMCs divide asymmetrically to produce a small triangular cell, the meristemoid, which serves as precursor of stomata guard cells and a larger cell referred to as the stomatal lineage ground cell (SLGC). The SLGC has the potential to directly differentiate into a lobed pavement cell or alternatively, to divide again asymmetrically to produce satellite meristemoids. All new meristemoids are oriented at least one cell away from an existing meristemoid according to the one-cell-spacing rule [3-7]. After up to three rounds of amplifying divisions, meristemoids mature into guard mother cells (GMC) acquiring the distinct rounded shape. A GMC divides symmetrically to generate two paired guard cells, which form the stomata pore. The genes responsible for GC specification and development have been characterised: the bHLH-type transcription factors (TFs) SPEECHLESS (SPCH), MUTE, 
and FAMA act sequentially to regulate formation of meristemoids, GMCs and GCs, respectively [8-10]. Alongside the afore-mentioned genes, another class of bHLH-type TFs, SCREAM/ICE1 and SCREAM2 redundantly affect the activities of SPCH, MUTE and FAMA through heterodimerization [11]. Previous studies have shown that $S P C H$ is required for cells to enter the stomatal cell lineage and to promote the amplifying divisions of the meristemoids $[9,10,12]$. Experiments utilising $S P C H$ promoter-reporter transcriptional fusions revealed that $S P C H$ is expressed in the developing leaf epidermis and persists in GMC and GCs throughout the lineage. However, the SPCH protein has only been detected in undifferentiated PDCs, MMCs and in young meristemoids, suggesting that $S P C H$ is regulated at the post-transcriptional level [9]. The activity of SPCH protein is negatively regulated by a signalling cascade, which includes secreted peptides EPIDERMAL PATTERNING FACTORS 1 and 2 (EPF1/2), leucine-rich repeat (LRR) receptor-like kinases ERECTA and TOO MANY MOUTHS (TMM) [3-5, 7, 13, 14]. The MITOGEN ACTIVATED KINASE (MAPK) genes act downstream of the LRR receptors and include YODA, $M K K 4 / M K K 5$ and MPK3/MPK6 [15-17]. Stimulation of MAPK results in SPCH phosphorylation and inactivation by proteasomal degradation $[1-3,15,18]$.

Several signals converge to regulate the stability of $\mathrm{SPCH}$ protein, including the phytohormone Brassinosteroid and $\mathrm{CO}_{2}[19,20]$. SPCH protein stabilization in protodermal cells is critical to trigger its transcriptional activity and consequent GC lineage entry. Among the direct targets of SPCH is the EPF2 gene which encodes a peptide that activates a regulatory feed back loop that promotes SPCH protein destabilization [21]. Therefore modulation of SPCH activity translates multiple environmental and endogenous developmental signals into different GC patterns $[8-10,22]$.

Besides bHLHs, other transcription factors may play an important role in GC specification. The DNA BINDING WITH ONE FINGER (DOF) proteins are an important class of transcriptional regulators in Arabidopsis thaliana comprising 37 members [11, 23]. These proteins have been shown to be involved in several aspects of plant development including growth, germination and abiotic stress response $[9,10,12,24]$. Also, DOF-type factors are implicated in cell cycle control $[9,25]$. In stomata development, DOFs have been hypothesized to play a role in GC maturation $[3-5,7,13,14,26,27]$. Recently the DOF transcription factor STOMATAL CARPENTER 1 (SCAP1) has been shown to directly regulate essential processes related to guard cell maturation and function. Mutants of scap1 display altered levels of transcripts of multiple genes directly involved in stomatal movement and furthermore are defective in some mechanical properties of the GC cell wall [28]. The potential role of SCAP1 in stomata patterning has not previously been investigated. In this study we provide evidence that SCAP1 plays a key role in GC patterning, in a manner that is temporally and spatially distinct from its role in GC maturation. We observed SCAP1 expression throughout the leaf lamina at early developmental stages, when primordia consist of only undifferentiated cells. Mutants of scap 1 had significantly reduced stomatal density and stomatal index compared with wild type. Conversely, over expression of SCAP1 resulted in increased stomatal density and stomatal index. Furthermore SCAP1 expression temporarily overlapped with the expression of several other genes that regulate stomatal patterning, consistent with SCAP1 playing a role in stomata patterning. Induction of SCAP1 activity using a glucocorticoid-based system resulted in repression of several early stomatal patterning genes including SPCH, MUTE and EPF2, and the ectopic production of GCs with altered spacing and morphology. In accordance with these phenotypes, detailed confocal microscopic analysis of marker lines on expanding leaf primordia revealed that high levels of SCAP1 correlated with an increase in the population of meristemoids as well as the number of undifferentiated PDCs. Our work thus provides evidence for a novel role for SCAP1 in stomatal patterning

\section{Results}

\section{SCAP1 expression in leaf precedes GC specification}

To further elucidate the role of SCAP1 in stomatal development we characterised a scap1 transposon insertion mutant publicly available in the Cold Spring Harbour collection. This allele (dubbed scap1-2) carries a gene trap construct, which permits endogenous patterns of expression of the trapped gene to be visualised via GUS staining. We characterised scap1-2 plants at different developmental stages and revealed two distinct patterns of gene expression during leaf development (Fig. 1). At early developmental stages (preceding GC formation) GUS staining was present throughout the emerging leaf primordia (Fig. 1a). At later developmental stages of primordia, levels of GUS staining were highest at the flanks of the lamina and much reduced in the midvein region (Fig. 1b). In mature organs (i.e. leaves and cotyledons) the GUS signal was mainly confined to maturing GCs (Fig. 1c). GC-specific SCAP1 expression was very faint in scap1-2 mutants compared with transgenic proSCAP1:GUS-GFP lines (Fig. 1f) (see below).

The scap1-2 mutant carries a GUS reporter gene in antisense orientation with respect to the SCAP1 open reading frame (Additional file 1 ). To verify that the GUS pattern observed in the scap1-2 allele reflects endogenous $S C A P 1$ promoter activity we fused a 2977 base pairs genomic region upstream of the SCAP1 coding sequence to GUS and GFP and generated independent Arabidopsis 

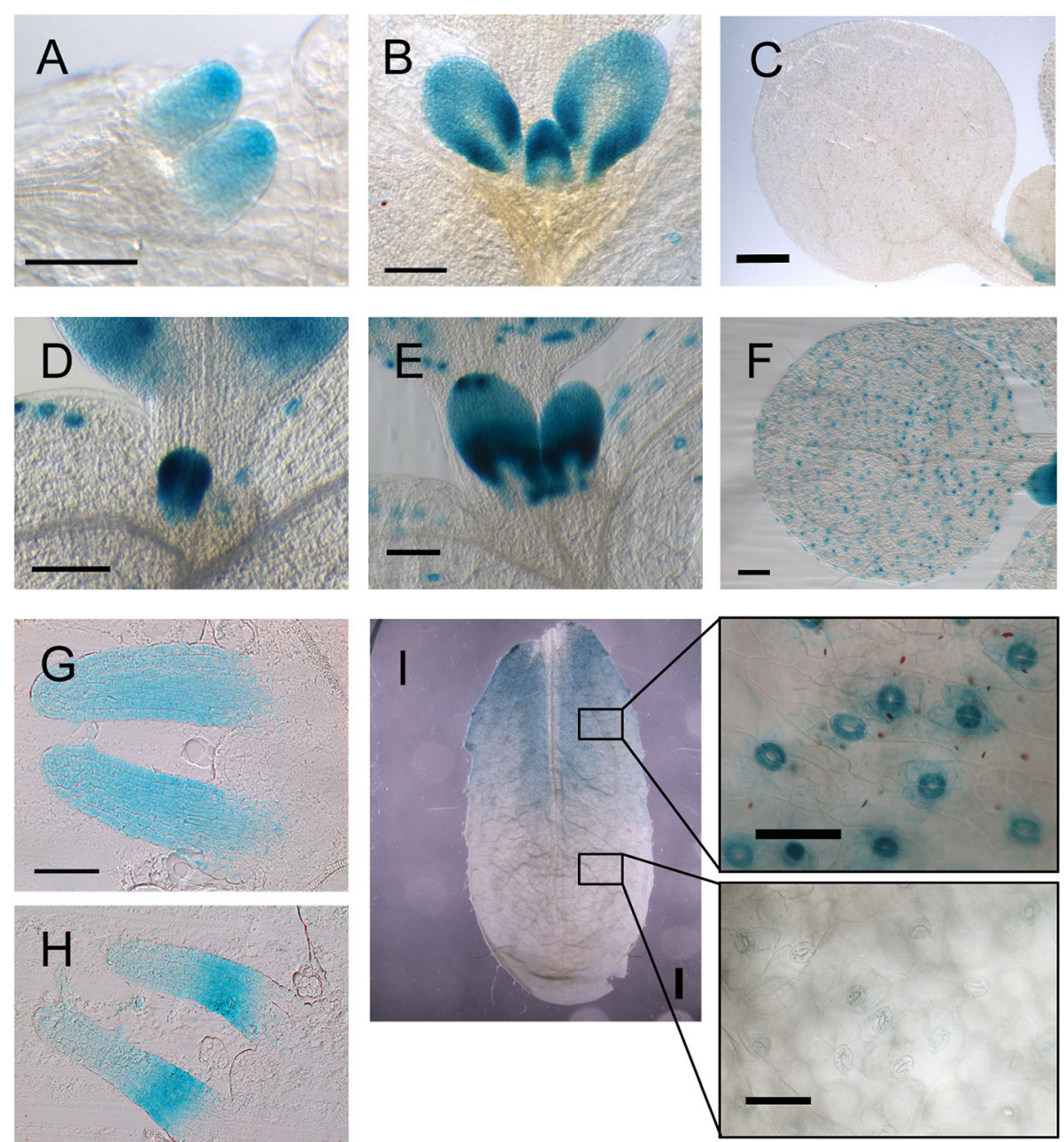

Fig. 1 SCAP1 expression patterns in emerging leaves. (a-c) GUS staining of the scap1-2 line and, (d-i), a representative proSCAP1:GUS-GFP transgenic line. Pictures were taken at different stages of leaf development from day $5(\mathbf{a}, \mathbf{d})$ to $7(\mathbf{b}, \mathbf{e})$. ( $\mathbf{g}, \mathbf{h})$ Transversal sections of leaf primordia of a proSCAP1:GUS-GFP seedling at day 5, (g) and 7 (h). (i) GCs specific GUS staining at different maturation stages in 3-week-old $6^{\text {th }}$ leaf. Bars $=50 \mu \mathrm{m}$ $(\mathbf{a}, \mathbf{b}, \mathbf{g}, \mathbf{h}) ; 500 \mu \mathrm{m}(\mathbf{c}, \mathbf{f}) ; 100 \mu \mathrm{m}(\mathbf{d}, \mathbf{e}, \mathbf{f}) ; 1 \mathrm{~mm}$ (i)

stable transformants. These transgenic plants (proSCAP1: GUS-GFP) displayed GUS activity in young leaf primordia which was similar to that observed in scap1-2 plants (Fig. 1d, e, f). At later stages of development, the pattern of GUS accumulation in the proSCAP1:GUS-GFP lines was broadly similar with that observed in scap1-2. Coincident with the expansion of leaf primordia, GUS staining gradually disappeared in the midvein region (Fig. 1e). In young leaf primordia, SCAP1 promoter activity appeared stronger in the proximal region of the leaf lamina. This observation was confirmed by analysing transverse sections of GUS stained proSCAP1:GUS-GFP plants. At early stages of primordium differentiation, the SCAP1 promoter was uniformly active in the mesophyll and the epidermis of leaf primordia (Fig. 1g). Subsequently we observed a sharp proximodistal gradient of GUS accumulation, with increased signal in the proximal part of the leaf primordium (Fig. 1h). SCAP1 expression was initially strong in GCs but tended to decrease in a distal to proximal gradient coincident with the maturation of GCs (Fig. 1i). These data reveal a previously undisclosed pattern of SCAP1 expression in early leaf development, which could suggest an additional role for SCAP1 alongside its already known function in GC maturation and function.

To gain insights in SCAP1 protein cellular localization we generated lines of Arabidopsis overexpressing SCAP1 $(n=15)$. The SCAP1 coding sequence was fused to the YELLOW FLUORESCENT PROTEIN (YFP) gene under the control of the constitutive promoter CaMV35S (pro35S:SCAP1-YFP). We anticipated that this construct would generate ectopic expression of SCAP1 throughout all plant tissues, however we were only able to observe 
YFP in a subset of plant tissues. The SCAP1-YFP protein-derived signal was absent in roots (Fig. 2a) whereas control plants overexpressing soluble YFP showed an ectopic signal in all tissues (Fig. 2f-i). We observed SCAP1-YFP accumulation in nuclei of mesophyll cells in young leaf primordia (Fig. 2b), while very little, if any SCAP1-YFP signal was observed in adjacent epidermal cells (Fig. 2c).
At later stages we observed SCAP1-YFP in GCs, which is consistent with the known function of SCAP1 in GC maturation (Fig. 2d). Detailed analysis of the epidermal layer of pro35S:SCAP1-YFP cotyledons revealed low levels of nuclear SCAP1-YFP protein in dividing (or recently divided) epidermal cells adjacent to differentiated GCs (Fig. 2e). In summary, the expression of SCAP1-YFP appeared restricted to the sub-epidermal layer in early
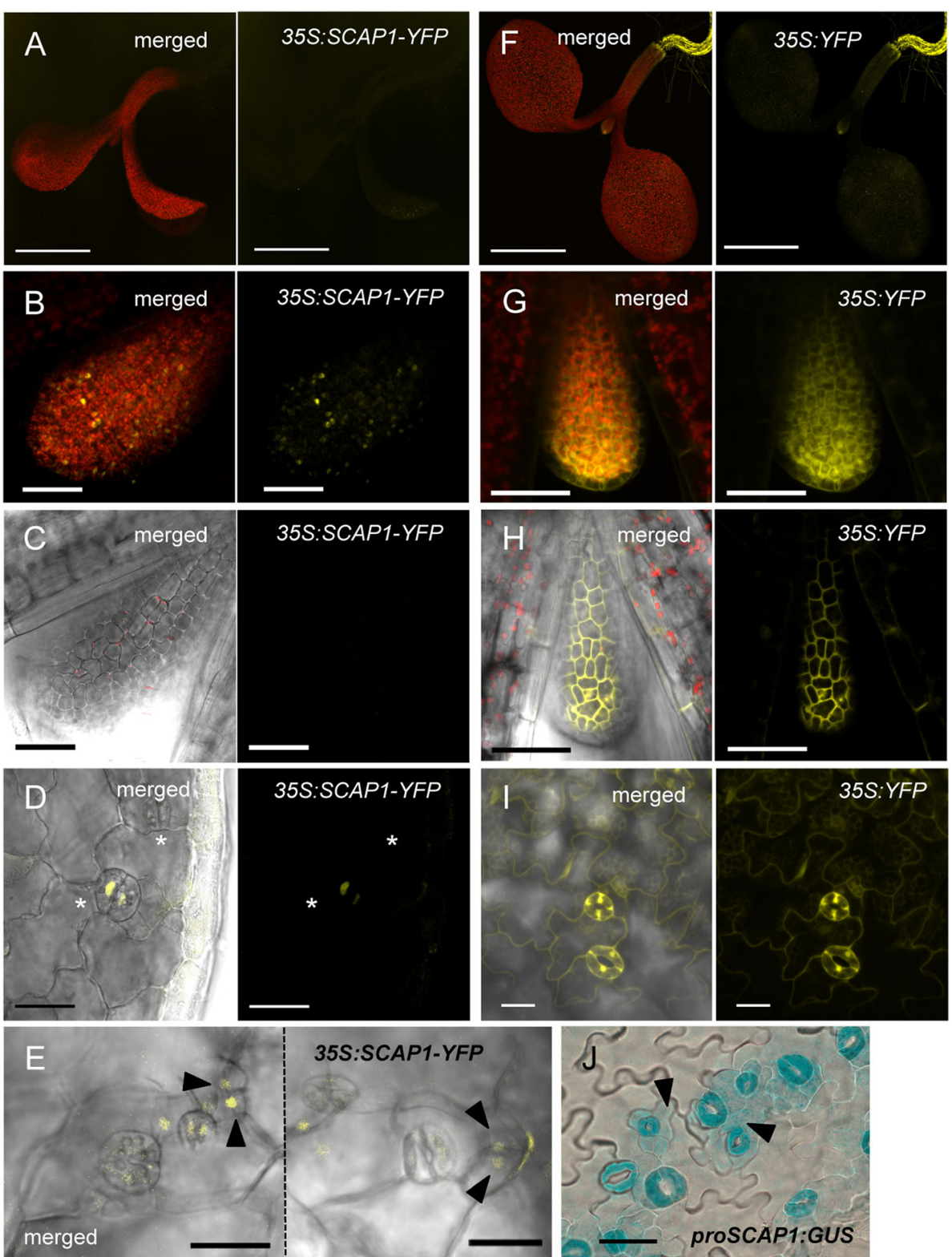

Fig. 2 SCAP1 protein differentially accumulates in plant tissues. (a-e) Confocal images of pro35S:SCAP1-YFP (35S:SCAP1-YFP) and, (f-i), pro35S:YFP (35S:YFP) plants at different stages. (a, f) Whole seedling. (b, g) First leaf primordia (5 das). (c, h) Epidermis of the first leaf primordia (5 das). (d, i) GCs in a cotyledon (7 das). (e) Epidermis of cotyledons (7 das). Asterisks mark epidermal cells, arrows heads mark dividing cells. Images $\mathbf{a}, \mathbf{b}, \mathbf{f}$ and $\mathbf{g}$ are the sum of all the $z$ stacks obtained across the entire thickness of the sample. Images $\mathbf{c}-\mathbf{e}$ and $\mathbf{h}-\mathbf{i}$ are the sum of those $z$ stacks corresponding to the epidermis. Bars $=1 \mathrm{~mm}(\mathbf{a}, \mathbf{f}) ; 50 \mu \mathrm{m}(\mathbf{b}-\mathbf{e}, \mathbf{g}-\mathbf{i})$. SCAP1-YFP/YFP protein signal is shown in yellow, autofluorescence (chlorophyll) in red. (j) Cotyledon epidermis of GUS stained proSCAP1:GUS-GFP plants (7 das). Bars $=100 \mu \mathrm{m}$ 
leaf primordia and only at later stages of leaf development the expression become visible in mature GCs and adjacent cells. The pattern of SCAP1 protein accumulation at later stages is similar to the domain where the SCAP1 promoter was transcriptionally active as shown by GUS staining of cotyledons of proSCAP1:GUS-GFP plants (Fig. 2j). We conclude that the SCAP1 protein is subject to a strong post-transcriptional regulation and that the site of SCAP1 protein accumulation only partially overlaps with the pattern of SCAP1 gene expression.

\section{SCAP1 regulates $\mathrm{GC}$ development}

The scap1-2 allele was likely a null since it did not produce any detectable full-length SCAP1 transcript (Additional file 1). To further investigate the role of SCAP1 in stomatal development we compared the number of GCs in adult leaves of scap1-2 mutants with that of wild type (ecotype Landsberg, Ler). In scap1-2 stomatal density is reduced (Fig. 3a) but this was not reflected in a reduction of stomatal index since scap1-2 plants also have a significant reduction in pavement cells compared to wild type (Fig. 3a, Additional file 2). To confirm these observations we generated two independent artificial microRNA (amiRNA1 and 2) constructs specifically targeting SCAP1 in wild type (ecotype Columbia, $\mathrm{Col})$. We isolated sixteen and fourteen independent T1 lines for amiRNA1-SCAP1 and amiRNA2-SCAP1, respectively and confirmed that $\mathrm{T} 2$ lines had reduced levels of SCAP1 transcript compared to wild type (Additional file 2). Downregulation of SCAP1 did not produce any obvious phenotypic effects on overall plant morphology, similar to scap1-2 plants. Closer observations revealed that leaves of segregating T2 amiRNA-SCAP1 knock-down independent lines produced significantly fewer GCs than wild type (Additional file 2). In homozygous T3 amiRNA-SCAP1 lines we observed a general reduction in cell density, analogous to the result observed in scap1-2, and also a reduction in stomatal index. Taken together these results suggest that SCAP1 plays a role in GCs specification in addition to its role in cell division (Fig. 3b, f).

To determine whether overexpression of SCAP1 is sufficient to alter GC development we analysed the phenotypes of the aforementioned pro35S:SCAP1-YFP lines. We observed $\mathrm{T} 1$ individuals with altered phenotypes ranging in severity from strong to mild (Fig. 3c). Plants classified as strong over-expressors of SCAP1 (60\%) exhibited numerous developmental defects including reduced germination, slow and stunted growth, upward-curling leaves and sterility. A second phenotypic class (40\%) displayed a less severe phenotype, exhibiting reduced growth compared to wild type at the seedling stage. In transgenic lines with intermediate phenotype, defects appeared to recover at later stages of development so that these lines were eventually comparable in final size and leaf area to wild type.
Given the strong phenotypic abnormalities in strong SCAP1-YFP overexpressing lines, we carried out our analyses on intermediate lines, which are more comparable to wild type in terms of plant morphology. Lines with intermediate levels of pro35S:SCAP1-YFP had an increased number of both GCs and PCs in true leaves compared to wild type and this was accompanied by an overall increase in stomatal index (Fig. 3d, f and Additional file 2). The epidermal phenotype of pro35S:SCAP1-YFP plants was characterised in more detail by crossing to a $\mathrm{GC}$-specific reporter line carrying proAtMYB60:GUS [29] which allowed us to detect subtler GC patterning defects. The cotyledons of pro35S:SCAP1-YFP showed gross alterations in stomata spacing as shown by the presence of massive clusters of GCs which were located at the edges of the cotyledon, especially on the adaxial surface (Fig. 3e). Interestingly, no clusters of GCs were detectable in true leaves of pro35S:SCAP1-YFP plants. Furthermore, based on GUS detection, over expression of SCAP1 did not confer guard cell identity to every cell type, nor was it able to induce stomata production in the cotyledon mesophyll cells (Fig. 3e). Thus, SCAP1 also plays an important role in determining $\mathrm{GC}$ spacing, at least in cotyledons.

To confirm these observations we generated a second gain-of-function allele of SCAP1 in which constitutively expressed SCAP1 is fused to the GLUCOCORTICOID RECEPTOR (pro35S:SCAP1-GR) [30]. In this inducible system the fusion protein is normally localised in the cytosol but can shuttle to the nucleus upon application of DEXAMETHASONE (DEX) to trigger a rapid SCAP1dependent transcriptional activation [30]. Prior to induction, plants of pro35S:SCAP1-GR were phenotypically indistinguishable from the wild type (Fig. 4e), despite accumulating high levels of SCAP1-GR transcript (Additional file 3). pro35S:SCAP1-GR seeds did not germinate on media supplemented with DEX, suggesting high levels of SCAP1 could inhibit germination. Therefore we grew pro35S:SCAP1-GR seeds on DEX-free media and transferred seedlings 5 days after sowing on media supplemented with DEX or a mock solution. Twenty days following transfer to DEX pro35S:SCAP1-GR plants produced similar morphological alterations previously observed in strong pro35S:SCAP1-YFP transgenic plants (Fig. 4g). In contrast, DEX treatment had no significant morphological effects in control plants (Fig. 4c).

To further investigate the epidermal phenotype of SCAP1-GR plants we analysed the pattern of GUS distribution in pro35S:SCAP1-GR proAtMYB60:GUS double hemizygous plants. Microscopic analysis of untreated pro35S:SCAP1-GR proAtMYB60:GUS plants revealed GCs cluster in both cotyledons and leaves although these clusters were generally made of few GCs (Fig. 4f and Additional file 3). Also, pro35S:SCAP1-GR proAtMYB60: GUS plants frequently presented unpaired GCs as well as 
A

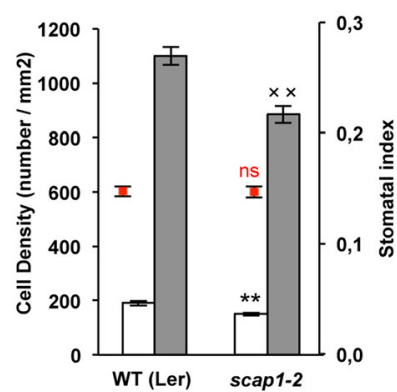

C

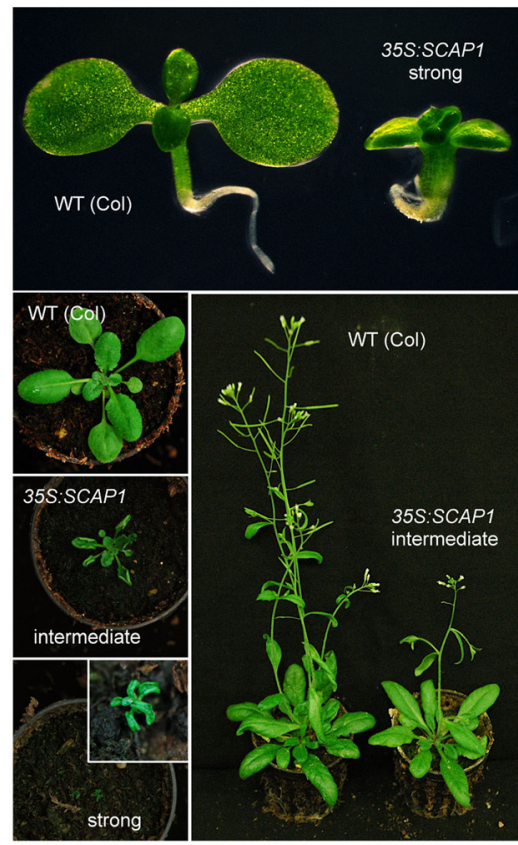

B

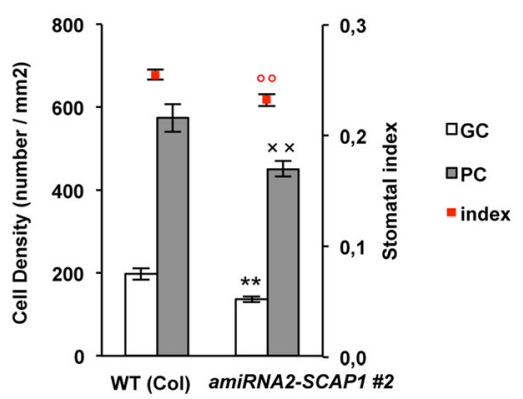

D

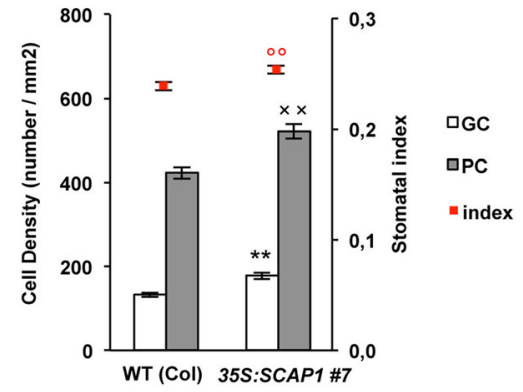

$\mathrm{E}$

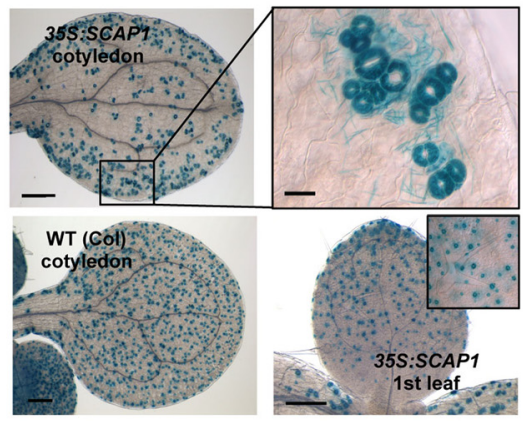

$\mathrm{F}$

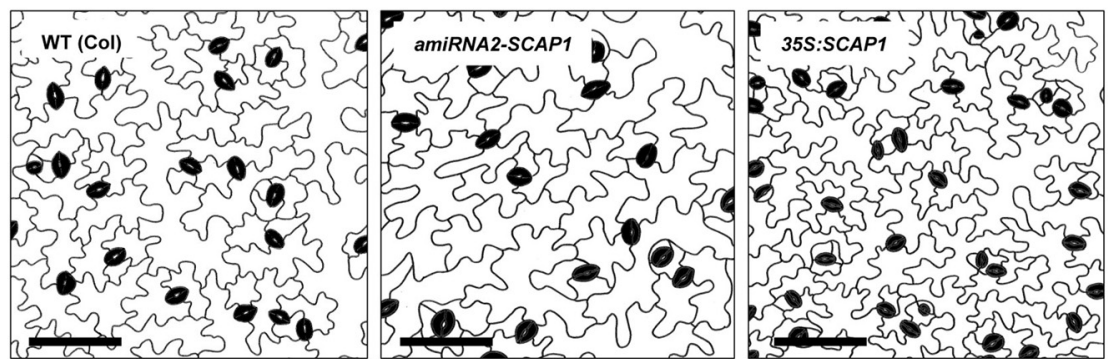

Fig. 3 SCAP1 controls GCs development. (a) Number of Guard cells (GC), pavement cells (PC) and stomatal index in wild type (Ler), and scap1-2 mutants and, (b), wild type (Col) and T3 homozygous pro35S:amiRNA2-SCAP1 (amiRNA2-SCAP1, line \#2). (c) Morphological alterations observed in pro35S:SCAP1-YFP (35S:SCAP1) lines at different developmental stages (seedlings, rosette, bolting plants). (d) Number of guard cells (GC), pavement cells (PC) and stomatal index in wild type (Col) and a T3 homozygous pro35S:SCAP1-YFP (35S:SCAP1) intermediate line (line \#7). (e) GUS staining of double proAtMYB60:GUS pro35S:SCAP1-YFP (35S:SCAP1) or single proAtMYB60:GUS (WT Col) hemizygous lines. Shown are mature cotyledons (inset, higher magnification of a representative cotyledon area) and the first leaf of 10 days old seedlings. Bar $=200 \mu m$ (inset, $25 \mu \mathrm{m}$ ). (f) Representative abaxial epidermal phenotype of the $6^{\text {th }}$ expanded leaf of wild type (Col), pro35S:amiRNA2-SCAP1 (amiRNA2-SCAP1, line \#2) and pro35S:SCAP1-YFP (35S:SCAP1, line \#7) mutants. Guard cells are false coloured in black. Bar $=50 \mu \mathrm{m}$. In $\mathbf{a}, \mathbf{b}, \mathbf{d},\left({ }^{* *}\right),\left({ }^{(X)}\right)$ and $\left({ }^{\circ}\right)=P<0.01$ (two tails T Student test) for comparisons between the wild type and the mutant alleles for GC, PC cell density or stomatal index, respectively. ns = not significant. Error bars $=$ Standard Error

clusters of meristemoid cells adjacent to GCs (Fig. 4f and Additional file 3). DEX treated pro35S:SCAP1-GR proAtMYB60:GUS plants, showed an even stronger phenotype in stomata patterning compared with the untreated control as we observed an overproduction of GCs in true leaves, which were grouped in extensive 

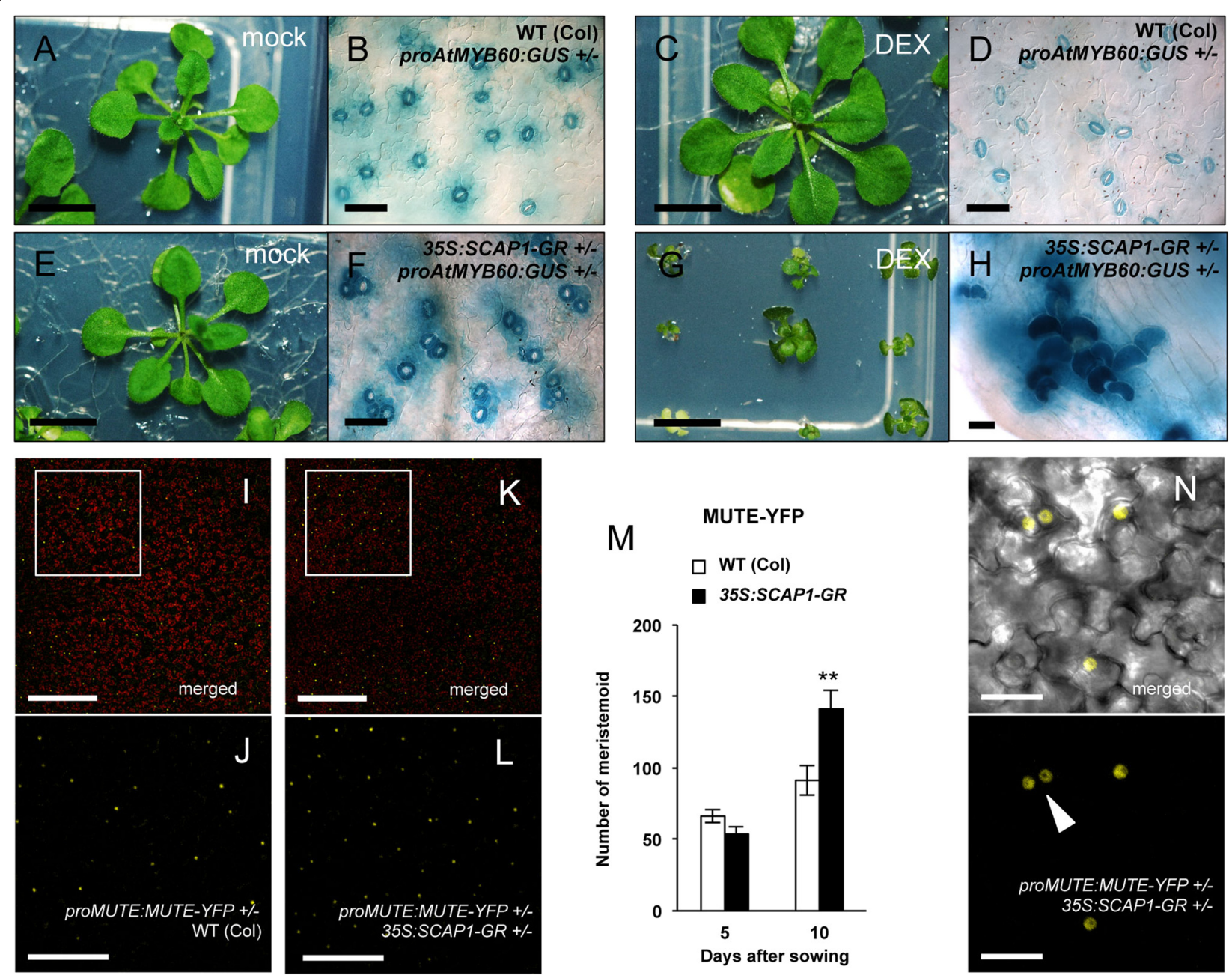

Fig. 4 SCAP1 affect stomata spacing and induce meristemoid production. (a-d) Morphological alterations observed in 4-weeks old wild type (Col) or (e-h) pro35S:SCAP1-GR (35S:SCAP1-GR) plants grown in presence of DEX (c-d and $\mathbf{g}-\mathbf{h}$ ) or mock (a-b and $\mathbf{e}-\mathbf{f})$. (f, $\mathbf{h}$ ) GUS staining of double proAtMYB60:GUS pro35S:SCAP1-GR or (B, D) single proAtMYB60:GUS hemizygous plants. (i-j) Confocal images of MUTE-YFP fusion proteins in hemizygous proMUTE:MUTE-YFP or ( $\mathbf{k}-\mathbf{I}$ and $\mathbf{n}$ ) double hemizygous proMUTE:MUTE-YFP pro35S:SCAP1-GR transgenic plants. Insets $(\mathbf{j}$ and $\mathbf{I})$ are higher magnification of the areas shown in (i) and (k), respectively. White arrowhead in (n) indicates two adjacent meristemoid. (m) Number of epidermal cells accumulating MUTE-YFP protein in hemizygous proMUTE:MUTE-YFP or double hemizygous proMUTE:MUTE-YFP pro35S:SCAP1-GR plants at different developmental stages ( 5 and 10 das). Shown is the average number of observable nuclei expressing MUTE-YFP in epidermal cells of 10 independent $1^{\text {st }}$ leaf primordia. Note that at stage 5 das, numbers refer to the entire primordium while at 10 das numbers refer to an area of $562 \mathrm{~mm}$. Error bars $=$ Standard Error. ${ }^{* *}=\mathrm{P}<0.01$ two tails T Student test. Bars $=1 \mathrm{~mm}(\mathbf{a}, \mathbf{c}, \mathbf{e}, \mathbf{g}) ; 200 \mu \mathrm{m}(\mathbf{i}, \mathbf{l}) ; 100 \mu \mathrm{m}(\mathbf{j}, \mathbf{m}) ; 50 \mu \mathrm{m}(\mathbf{b}, \mathbf{d}, \mathbf{f}) ; 20 \mu \mathrm{m}(\mathbf{h}, \mathbf{o})$

clusters (Fig. 4h). Also in this case, GUS detection revealed that clusters were frequently made of unpaired GCs (Fig. 4h).

To identify whether the altered stomata patterning of pro35S:SCAP1-GR could depend on increased number of cells entering the stomatal lineage we generated double hemizygous proMUTE:MUTE-YFP pro35S:SCAP1-GR plants which allowed us to visualise meristemoid cells. Even in the absence of DEX, at the later stages of primordium development pro35S:SCAP1-GR proMUTE: $M U T E-Y F P$ plants displayed an increased number of meristemoids compared to control hemizygous proMUTE: MUTE-YFP plants (Fig. 4i to l). A closer inspection of the epidermis revealed that in pro35S:SCAP1-GR proMUTE: MUTE-YFP meristemoid cells often did not follow the correct spacing and were close to each other (Fig. 4n). Taken together SCAP1 appears to regulate different aspects of stomata development, including stomata number, distribution and spacing.

Effects of SCAP1 on stomatal patterning gene expression The early activation of SCAP1 in leaf primordia coupled with its role in stomata development led us to hypothesise a genetic interaction between SCAP1 and genes that regulate stomatal patterning. Two genes, $S P C H$ and $E P F 2$ that are required for early stomatal patterning are 
expressed in the protodermal cells of leaf primordia. To determine the timing of SCAP1 activation with respect to stomata early patterning genes we sampled primordia of leaves one and two from seedlings at different time points, representative of different stages of leaf development. Transcript abundance of SCAP1, SPCH and EPF2 peaked at 7 days after sowing and subsequently decreased during the next 3 days (Fig. 5a). At around 12 days after sowing, SCAP1 expression levels reactivated, presumably in relation to GC formation in the maturing leaf (Fig. 5a). To test if SCAP1 expression is dependent on $S P C H$, we crossed scap1-2 (Ler) with spch-4 (Col) mutants to obtain homozygous spch mutants carrying a transposon tagged version of SCAP1. Of 26 spch homozygous plants, two displayed GUS staining that was similar in terms of pattern of expression to wild type $S P C H$ plants. The reduced frequency of this genotype could be due to genetic linkage since $S P C H$ and SCAP1 are physically close on chromosome 5. $S P C H$ is thus not required for the early SCAP1 activation (Fig. 5b), consistent with previous studies indicating that SCAP1 was not a high-confidence SPCH target [21].

We next measured transcript accumulation of early stomatal patterning genes in plants with different dosage of SCAP1. Transcript levels of SPCH, EPF2, MUTE and FAMA were analysed at 7 DAS when levels of SCAP1, $S P C H$ and EPF2 expression are at their peak in the wild type (Fig. 5a). In loss-of-function scap1 mutant plants we detected no significant changes in transcript levels of any of the genes analysed compared with the wild type (Fig. 5c and additional file 4). If SPCH and SCAP1 genetically interact we might predict that increased GC production in pro35S:SCAP1-YFP plants would be reflected in an increased level and/or activity of positive regulators of stomatal production, or alternatively down regulation of negative regulators. To determine if this is the case we analysed transcript levels of SPCH, EPF2, MUTE and FAMA in the pro35S:SCAP1-YFP over expression line. Our analysis confirmed that this transgene conferred around 100-fold increase in SCAP1 transcript accumulation when compared to wild type (Fig. 5c). Analysis at 7 DAS revealed no significant difference in transcript levels of either MUTE or FAMA compared to wild type (Fig. 5c). However, we noticed a down regulation of EPF2 and, marginally, SPCH as compared to the wild type (Fig. 5c).

To confirm these observations we analysed the stomatal patterning genes in pro35S:SCAP1-GR plants after a short DEX induction. We first tested the ability of SCAP1:GR

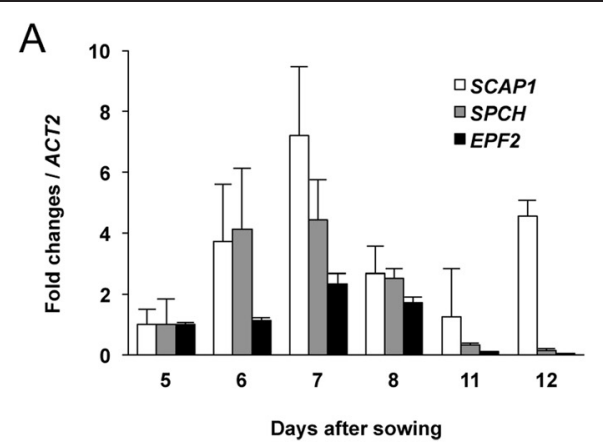

B
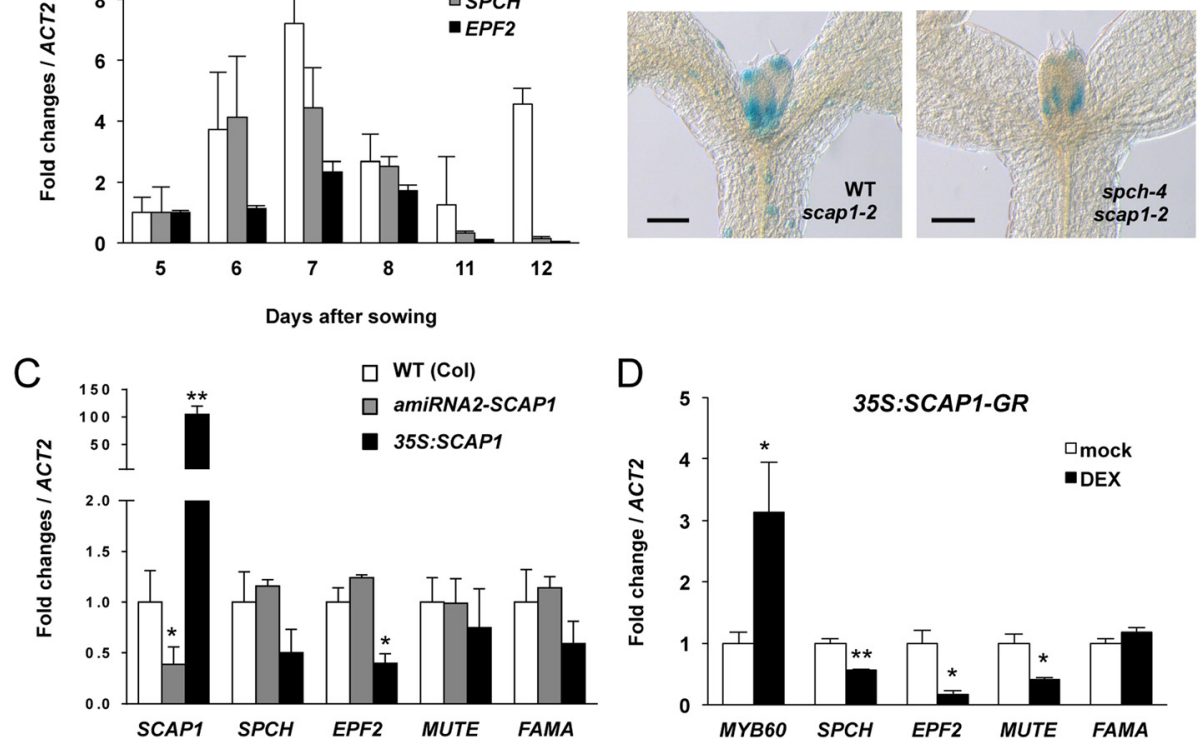

Fig. 5 Role of SCAP1 on stomatal genes transcript accumulations. (a) Pattern of SCAP1, SPCH and EPF2 transcript accumulations determined by quantitative PCR in manually dissected first two leaf primordia of wild type (Col) seedlings at different days after sowing. Values represent the mean of three biological replicates (30 leaves / replica). (b) GUS staining of scap 1-2 in wild type or spch-4 mutant background in 5 day old seedlings. Bar $=100 \mu \mathrm{m}$. (c) Pattern of SCAP1, SPCH, EPF2, MUTE and FAMA transcript accumulations determined by quantitative PCR in manually dissected first two leaf primordia of 7 days-old wild type (Col), pro35S:amiRNA2-SCAP1 (amiRNA2-SCAP1) and pro35S:SCAP1-YFP (35S:SCAP1) plants. Values represent the mean of three biological replicates (30 leaves / replica). (d) Pattern of AtMYB60, SPCH, EPF2, MUTE and FAMA transcript accumulations determined by quantitative PCR in 10 days-old pro35S:SCAP1-GR (35S:SCAP1-GR) plants treated by spraying with DEX (or mock) and the whole seedlings were sampled at eight hours after treatment. Values represent the mean of two biological replicates. In all quantitative PCR ACTIN (ACT2) was used for normalization. In $\mathbf{c}$ and $\mathbf{d}^{* *}=\mathrm{P}<0.01$ and ${ }^{*}=\mathrm{P}<0.05$ and two tails T Student test. Error bars $=$ standard deviation 
protein to activate expression of its known target gene AtMYB60 [28]. Indeed, scap1 loss of function mutants displayed reduced levels of AtMYB60 accumulation compared with wild type (Additional file 3). Conversely, compared to wild type plants, pro35S:SCAP1-GR plants showed up-regulation of AtMYB60 after DEX treatment (Fig. $5 \mathrm{~d}$ and Additional file 3). These data indicate that SCAP1-GR protein retains its biochemical function in the context of transcriptional regulation.

Under similar conditions, eight hours after induction we observed a strong downregulation of the negative stomatal regulator EPF2 (Fig. 5d and Additional file 4). Such EPF2 downregulation became detectable in DEX treated compared to mock treated plants after four hours and was maintained throughout our experiment (Additional file 4). Besides EPF2 we also observed a general downregulation of $S P C H$ transcript levels and its direct target gene MUTE, but not FAMA (Fig. 5d). As a control, DEX treatment on wild type plants had no effects in altering stomata patterning genes (Additional file 4). SCAP1 can therefore act both as a positive and negative transcriptional regulator. However as these experiments were performed on whole seedlings, they may not entirely revel the mode of action of SCAP1 during the early stages of leaf development.

\section{SCAP1 affects SPCH protein accumulation}

Constitutive expression of SCAP1 resulted in several developmental abnormalities, which could indirectly alter GC development. To avoid this potential problem, we analysed the effect of SCAP1 after rapid activation by DEX using the pro35S:SCAP1-GR line. We studied the pattern SPCH-GFP fusion protein accumulation in the primordia of the first leaf ( 5 das) through microscope confocal analysis by visualizing nuclear fluorescence of GREEN FLUORESCENT PROTEIN (GFP) in a proSPCH:SPCH-GFP line. At 5 das, we did not detect variations in the number of meristemoids, suggesting that SCAP1-GR expression did not yet produce detectable effects at this particular stage (Fig. $4 \mathrm{~m}$ ). We reasoned that by providing a short pulse of SCAP1 (through DEX applications) we could influence the competence of cells entering the stomata lineage (as estimated by the number of cells expressing $\mathrm{SPCH}$ ). We generated hemizygous proSPCH:SPCH-GFP proPIN3:PIN3-GFP pro35S:SCAP1-GR or hemizygous proSPCH:SPCH-GFP proPIN3:PIN3-GFP in a wild type Col background. The PIN3-GFP fusion protein was used as plasma membrane marker and allowed us to identify individual epidermal cells. In control double hemizygous proSPCH:SPCH-GFP proPIN3:PIN3-GFP plants no significant differences were found in the number of SPCH-GFP expressing cells following DEX treatment (Fig. 6b and c). Also, DEX treatment did not alter the average intensity of nuclear SPCH-GFP fluorescence, which rules out a general effect of DEX on SPCH-GFP protein accumulation (Fig. 6d). proSPCH:SPCHGFP proPIN3:PIN3-GFP pro35S:SCAP1-GR hemizygous lines showed no apparent defects in SPCH-GFP accumulation at this developmental stage (Fig. 6a to d). At 6 hours following DEX treatment, proSPCH:SPCH-GFP proPIN3: PIN3-GFP pro35S:SCAP1-GR plants showed a significant increase in the proportion of nuclei expressing SPCH-GFP protein (Fig. 6a, b and c). Furthermore, this was accompanied with a general increase in the mean nuclear GFP fluorescence intensity $\left(n>50\right.$ nuclei / $1^{\text {st }}$ leaf primordia for each genotype/treatment combination) (Fig. 6a, d). It is most likely that the increased nuclear GFP signals reflected increased SPCH-GFP protein since neither DEX treatment or SCAP1-GR alone caused variations in nuclear GFP accumulations (e.g. as a result of detachment of GFP from the membrane marker PIN3 or SPCH). Increased SPCH stabilisation in protodermal cells may thus contribute to stomata pattering alterations in SCAP1 over expressing plants.

\section{Discussion}

Previously SCAP1 was shown to control GC morphology and activity, a role coherent with its expression in developing and fully mature stomata [28]. Here we report an in-depth analysis of the spatio-temporal control of SCAP1 expression throughout leaf development. Our results indicate an early activation of SCAP1 expression in leaf primordia coinciding with the expression of genes controlling stomatal cell lineage and thus before GC differentiation $[6,7,10,31]$. This pattern of $S C A P 1$ gene expression is maintained in spch mutants demonstrating that SCAP1 early expression is independent of GC lineage specification. Besides transcriptional regulation, SCAP1 is regulated at the post-transcriptional level, as constitutively expressed SCAP1-YFP fusion did not accumulate in all plant tissues, despite high levels of expression. In leaf primordia where SCAP1 promoter is active in both epidermis and mesophyll, SCAP1-YFP protein was mainly observed in the mesophyll and in GCs. This observation may either suggest that the role of SCAP1 in GC development is indirect (e.g. to promote signals from the mesophyll cells to the epidermis [32-35] or that the activity of SCAP1 in the epidermis is tightly regulated as a result of rapid protein turn over. Therefore, SCAP1 protein may accumulate in the epidermis in some cell types or at certain stages. Future experiments involving the use of tissue/cell specific promoters to drive SCAP1 expression may help elucidate the precise cell/tissue-specific pattern of SCAP1 stabilization and provide clues as to the mode of action of SCAP1 in GC patterning.

A question emerging from our study is whether the role of SCAP1 in stomata patterning is direct or indirect. For example, changes in $\mathrm{CO}_{2}$ perceived by one leaf affect 


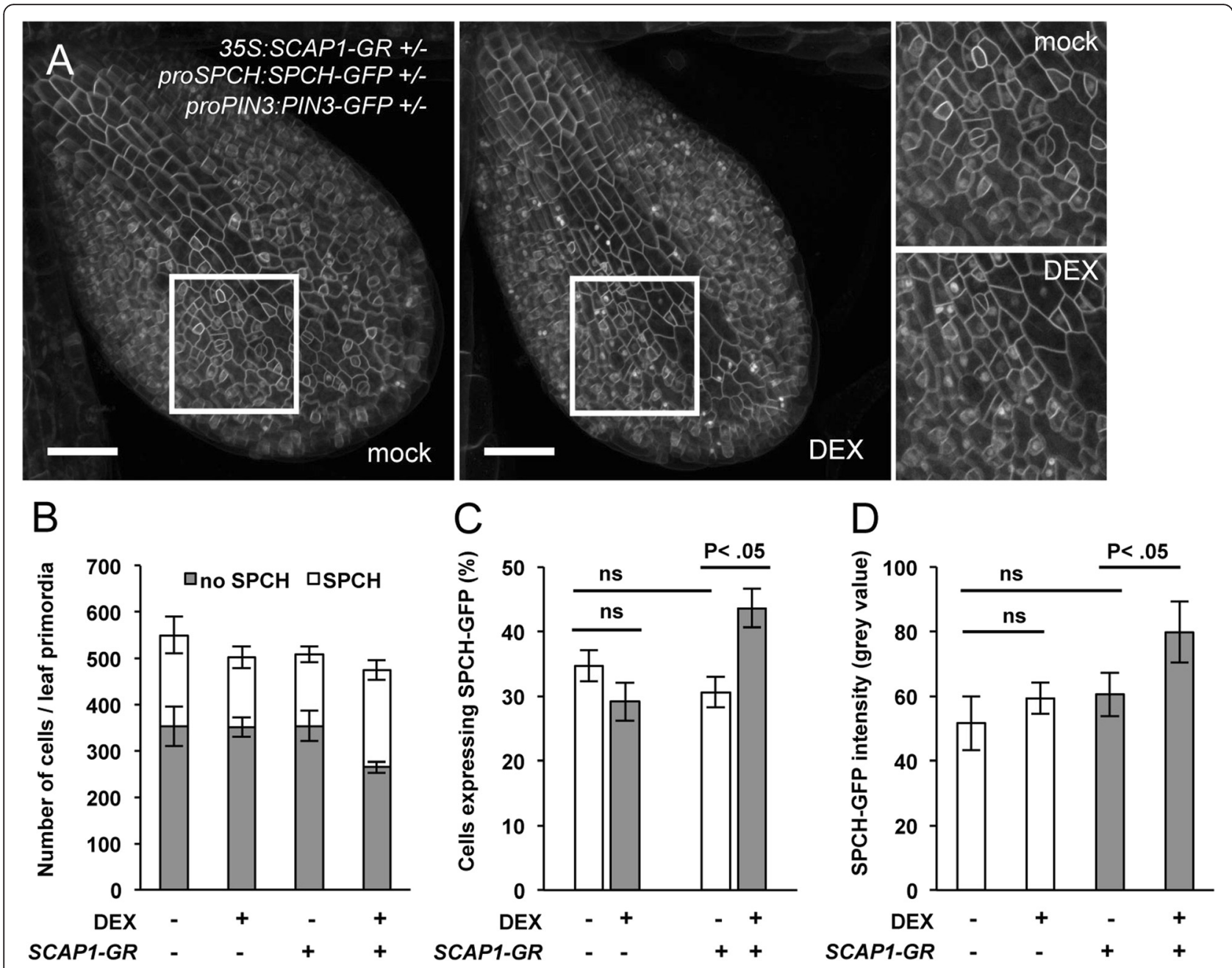

Fig. 6 SCAP1 promotes SPCH protein accumulation. (a) Representative picture of the $1^{\text {st }}$ leaf primordia of mock or DEX-treated triple hemizygous proPIN3:PIN3-GFP proSPCH:SPCH-GFP pro35S:SCAP1-GR transgenic plants. Insets show a portion of the primordia at higher magnification. PIN3-GFP fusion protein marks the plasma membrane of epidermal cells. SPCH-GFP fusion protein localises in nuclei of the epidermis. Scale bar $=500 \mu \mathrm{m}$. (b and $\mathbf{c}$ ) Quantification of nuclei accumulating SPCH-GFP in leaf primordia of mock (-) or DEX (+) treated plants. Double hemizygous proPIN3:PIN3-GFP proSPCH:SPCH-GFP were used as control (-) and compared to triple hemizygous proPIN3:PIN3-GFP proSPCH:SPCH-GFP pro35S:SCAP1-GR (SCAP1-GR, +). Bars represent the number of total epidermal cells forming the $1^{\text {st }}$ leaf primordium imaged at 5 das. White bars represent the number of nuclei containing SPCH-GFP. Grey bars are nuclei with no detectable SPCH-GFP. $n=6-8$ independent first leaf primordia. This experiment was performed twice with similar results. (c) Same data as (b) but shown as a percentage of nuclei expressing SPCH-GFP protein over the total number of cell composing leaf primordia (d) Quantification of the mean fluorescence intensity of nuclear SPCH-GFP protein in the indicated backgrounds/treatment. Data derived from the analysis of approx. 50 nuclei expressing SPCH-GFP in 6-8 independent first leaf primordia. In (c) and (d) P values denote a statistical significance in the number of SPCH-GFP nuclei or intensity of SPCH-GFP fluorescence, respectively, calculated with one-way ANOVA. NS = not significant. Error bars $=$ Standard Error

the patterning of GCs in subsequent leaves, implying the existence of a signalling network to optimize GC number and patterning according to environmental conditions [36-38]. Since scap1 mutants are impaired in GC function one could hypothesise that such alterations in stomata activity may affect global GC development. Although we cannot exclude this possibility, we also showed that SCAP1 overexpressing plants had GC alterations in embryonic tissues such as cotyledons (where we observed GCs cluster). SCAP1-GR plants also had increased stomata cells density in true leaves arguing in favour of a direct role of SCAP1 in stomata patterning. Furthermore, a detailed analysis of SCAP1-GR plants revealed a role of SCAP1 in both promoting GCs production and directing the spacing of meristemoid at the very early stages of stomatal cell lineage specification (Fig. 4i to $\mathrm{n}$ ). These observations are indicative of a role of SCAP1 in GC patterning which is independent of its general function in GC maturation. The accumulation of $S C A P 1$ transcript in young leaf primordia is consistent with an early role for SCAP1 in controlling GC development. SCAP1 could also play an additional role in the 
specification of GCs at later stages of leaf development, for example by controlling satellite meristemoid cells, since SCAP1 expression can be transiently detected in dividing (or recently divided) epidermal cells adjacent to differentiated GCs in older tissue (Fig. 2e).

The overall increased cell density of SCAP1 overexpressing plants is reminiscent of $S P C H$ overexpression or epf2 mutant plants $[6,7,9,15]$. Ectopic expression of SCAP1 could not initiate GCs development in the interior layers of cotyledons or leaves, suggesting that SCAP1 affects GCs production in conjunction with the known elements of stomatal cell lineage pathway (e.g. SPCH, MUTE and FAMA). Therefore, one attractive hypothesis arising from these observations is that SCAP1 participates in the same genetic pathway of GC development controlled by $S P C H$ and its regulators. Some evidence for this was provided by experiments which showed expression of $S P C H, E P F 2$ and SCAP1 temporarily overlapping during development. Mutants of scap1 are not defective in global $S P C H$ or EPF2 gene expression levels, although we have not tested the possibility that the spatial distribution of SPCH or EPF2 genes might be altered in scap1 mutant plants. Furthermore, the relatively weak epidermal phenotype of scap1 mutants might be masked by a yet unknown SCAP1-like function.

Not only did SCAP1 affect stomata number but also stomata spacing. Clusters of GCs were present in postembryonic tissues in pro35S:SCAP1-GR plants, a phenotype which was even further exacerbated upon DEX application. Surprisingly, this phenotype was not observed in pro35S:SCAP1-YFP plants (in which this spacing defects were confined to the cotyledons). The reasons why the SCAP1-GR fusion is more active than SCAP1-YFP is currently unknown. As SCAP1 accumulation is tightly controlled at the posttranscriptional levels, one possibility is that the GR moiety protects it from degradation.

SCAP1 overexpression caused EPF2 downregulation, which could account for spacing defects. The signalling peptides EPF2 acts early in the stomatal lineage controlling asymmetric cell division and thus regulating stomatal density [7]. Previously, comparably similar defects in stomata density and spacing were described in mutants of epf2, epf1 and tmm [3, 5-7, 31], or in transgenic plants overexpressing SPCH, MUTE or FAMA $[8,9,15]$. The $\mathrm{SPCH}$ protein directly binds and positively regulates the transcription of several stomatal patterning genes including EPF2, MUTE and TMM as well as its own transcription [21]. Our data indicates that some of the direct targets of $S P C H$ are negatively regulated by $S C A P 1$, suggesting a competition between $S P C H$ and SCAP1 for the regulation of these genes at transcriptional level. In this model, SCAP1 promotes stomata production and boosts cell divisions by enhancing $\mathrm{SPCH}$ protein accumulation possibly as a result of down regulation of EPF2 transcript. Whether this competition occurs in the same cell and/or is direct should be elucidated by further experiments.

\section{Conclusions}

Our results highlight a previously unappreciated role for SCAP1 in stomata development. We propose that SCAP1 is an essential component of a genetic pathway to finetune stomatal production in Arabidopsis. A key control mechanism of this loop could involve a SCAP1-mediated downregulation of EPF2 counteracting the previously demonstrated SPCH-mediated activation of EPF2 [21]. DOF-type factors have been proposed to play an important role in GC maturation and function based on an enrichment of a DOF binding motif in GC specific genes $[26,27]$. It would be interesting to test whether this observation can also be extended to genes involved in the early events of GC lineage specification. In this sense, SCAP1 may link GC patterning and function.

\section{Methods \\ Plant material and growth conditions}

In this study we used Arabidopsis thaliana ecotypes Columbia (Col) and Landsberg erecta (Ler). Seeds were germinated and plants grown in a controlled-environment cabinet at a temperature of $20{ }^{\circ} \mathrm{C}$ to $23{ }^{\circ} \mathrm{C}, 65 \%$ relative humidity, under long day conditions ( $16 \mathrm{~h}$ of light $/ 8 \mathrm{~h}$ of dark). Light was cool-white fluorescent tubes (Osram; Sylvania) at a fluency of 120 to $150 \mu \mathrm{E}$ (photosynthetically active radiation). The scap1-2 allele is a transposon insertion (line GT-23689, Ler background) obtained from the Cold Spring Harbour gene trap collection (http:// genetrap.cshl.edu). The spch-4 knock out allele and the proSPCH:SPCH-GFP proPIN3:PIN3-GFP and proMUTE: MUTE-YFP lines were previously detailed [9, 12, 39]. The proAtMYB60:GUS line (Col background) was previously described [29]. The proSCAP1:GUS-GFP, pro35S: amiRNA-SCAP1, pro35S:SCAP1-YFP and pro35S:SCAP1$G R$ lines were generated in this study in wild-type Col background except for the proSCAP1:GUS-GFP which was in the Ler background. Transgenic lines were obtained using the floral dipping method [40]. Transgenic seedlings were selected on Murashige and Skoog (MS) media with kanamycin $(50 \mu \mathrm{g} / \mathrm{ml})$ (pro35S:SCAP1-GR) or Basta $(25 \mu \mathrm{M})$ (proSCAP1:GUS-GFP, pro35S:amiRNASCAP1, pro35S:SCAP1-YFP and pro35S:SCAP1-GR). For each construct several $\mathrm{T} 1$ independent lines were generated and single insertion transgenic plants were isolated based on the segregation of resistance genes. Independent homozygous T3 lines analysed in this study are : pro35S:SCAP1-YFP (\#4 and \#7), pro35S:amiRNA-SCAP1 (amiRNA1-2, amiRNA2-2 and amiRNA2-5), proSCAP1: GUS-GFP (\#8 and \#2), pro35S:SCAP1-GR (\#34 and \#26). 
Glucocorticoid applications were done by administering a solution of DEX (13 $\mu \mathrm{M}$ DEX, $0.01 \%(\mathrm{v} / \mathrm{v})$ Tween 20) either by spraying (for expression analysis) or by soaking seedlings in a MS medium containing DEX (for confocal and GUS experiments). Stratified pro35S:SCAP1-GR seeds were germinated on MS plates for 5 to 7 days before spraying with DEX or mock treated or transferred in phytatray (Sigma-Aldrich) on MS media containing DEX for prolonged glucocorticoid treatment.

\section{Molecular cloning}

To generate the proSCAP1:GUS-GFP construct a $2977 \mathrm{bp}$ region upstream of the SCAP1 start codon was amplified from genomic DNA by PCR with oligos attB1-SCAP1 and attB2-SCAP1 which contain the $A t t B$ adaptors for Gateway-mediated cloning. The PCR product was cloned into $p D O N R 207$ and subsequently transferred to pBGWFS7 destination vector [41] according to the guidelines detailed in the Gateway protocol (Life Technologies). The pro35S:amiRNA-SCAP1 constructs were engineered as detailed in http://wmd3.weigelworld.org [42] with primers I, II, III and IV. The PCR products containing SCAP1-specific amiRNA were cloned in the pENTRDTOPO vector (Life Technologies) and transferred to the destination vector pEarleyGate 100 [43] via LR-mediated recombination. To generate the pro35S:SCAP1-YFP, the SCAP1 open reading frame (without stop codon) was amplified by PCR from Arabidopsis DNA, with primers SCAP1-Fw, SCAP1-Re2 and cloned into the pENTR-D TOPO vector (Life Technologies) and recombined with the Gateway destination vector pEarleyGate 101 [43]. The DEX-inducible SCAP1 construct (pro35S:SCAP1-GR) was kindly provided by the RIKEN Plant Functional Genomic Minami Matsui lab. Sequences of the primers are detailed in Additional file 5.

\section{Genotyping and transcript analysis}

Sequences of the primers used for genotyping are provided in Additional file 5. Total RNA was extracted with TRIzol reagent following the manufacturer's instructions (Life Technologies). The first-strand cDNA was synthesized with 500 ng of total RNA using SuperScript VILO Reverse Transcriptase kit (Life Technologies). Quantitative real-time PCR was performed with Fast SYBR Green Master Mix (Applied Biosystems), and amplification was real-time monitored on a 7900 HT Fast Real-Time PCR system (Applied Biosystems). Changes in gene expression were calculated relative to $A C T 2$ using the $\triangle \triangle \mathrm{Ct}$ method [44]. The qPCR primers to detect SCAP1, AtMYB60, $S P C H, E P F 2, M U T E, F A M A$ and ACTIN transcripts are detailed in Additional file 5.

\section{$\beta$-glucuronidase (GUS) histochemical assay and Histological procedures}

GUS staining was performed as previously described [26]. Depending on the experiment, incubation time was for 4 to $12 \mathrm{~h}$ at $37^{\circ} \mathrm{C}$. For detection of GUS staining in thin resin sections, after staining, samples were dehydrated in $70 \%(\mathrm{v} / \mathrm{v})$ ethanol, post-fixed over night at $4{ }^{\circ} \mathrm{C}$ in FAE $(50 \%[\mathrm{v} / \mathrm{v}]$ ethanol, $5 \%[\mathrm{v} / \mathrm{v}]$ formaldehyde, $10 \%$ $[\mathrm{v} / \mathrm{v}]$ acetic acid), and further dehydrated in a series of $85 \%, 95 \%$ and $100 \%(\mathrm{v} / \mathrm{v}) \mathrm{EtOH}$ and embedded in Technovit 7100 resin according to the manufacturer's instructions (Heraeus Kulzer). Samples were sectioned with a microtome fitted with a stainless steel blade to a $7 \mu \mathrm{M}$ thickness.

\section{Microscopy and quantitative analysis of fluorescence emission}

For analysis of the stomatal pattern, the $6^{\text {th }}$ expanded leaves of one-month-old plants (displaying an inflorescence of about 3-4 cm) were incubated in $70 \%$ ethanol. The epidermis of the abaxial side was peeled and examined under a transmission light microscope (DM2500, Leica). For determining the mean stomatal index and density, one square area $\left(0.2 \mathrm{~mm}^{2}\right)$ of a leaf region was microphotographed and scored for cell parameters. Care was used to select a similar leaf region from the $6^{\text {th }}$ leaf from at least 12 independent plants for each genotype in independent experiments. For confocal laser scanning microscopy, the abaxial side of first leaf primordia of 5-day-old seedlings expressing GFP- or YFP-tagged proteins were analysed under a Leica TCS SP5 confocal microscope. Fluorochromes were excited using an Argon laser (488 nm and $514 \mathrm{~nm}$ excitation for GFP and YFP, respectively) and emission collected at a $500-570 \mathrm{~nm}$ and $525-600 \mathrm{~nm}$ for the GFP and YFP, respectively. When comparing independent samples, the acquisition parameters (including z-step size) were maintained constant to enable measurement of GFP intensity in different primordia and different treatments. Fluorescence intensity of nuclear SPCH-GFP protein was analysed with ImageJ software (http://imagej.nih.gov/ij/). GFP fluorescence intensity was measured from individual nuclei (at least 50 for each primordium). A region of interest (ROI) tool was superimposed to selected nuclei so to include the largest possible nuclear area in single optical plane (z stack). An identical ROI size was used to process all the images so to minimise detection of background fluorescence.

\section{Additional files}

Additional file 1: Characterisation of the scap1 mutant allele. (A) Schematic representation of the SCAP1 loci. Grey boxes represent amiRNAs target regions and triangles represent transposon genomic insertion points for scap 1-2. (B) Reverse Transcriptase-PCR analysis of SCAP1 in wild type 
(Ler) and scap1-2 plants. Total RNA was isolated from 2-week-old seedlings and PCR was conducted for 35 cycles. Actin was used as a positive control and amplified for 25 cycles. (JPG $97 \mathrm{~kb}$ )

Additional file 2: SCAP1 affects GCs development. (A) Representative abaxial epidermal phenotype of a $6^{\text {th }}$ expanded leaf of wild type (Ler) and scap 1-2 mutants. Guard cells are false coloured in black. Scale bar $=50 \mu \mathrm{m}$. (B) Pattern of SCAP1 transcript accumulation determined by quantitative $P C R$ in mature leaves in independent T1 BASTA resistant pro355:amiRNA-SCAP1 (amiRNA-SCAP1) transgenic lines, compared with wild type (Col-0). ACTIN (ACT2) was used for normalization. Values represent the mean of two technical replicates. Error bars $=$ standard deviation. (C) Number of Guard cells (GC), pavement cells (PC) and stomatal index in wild type (Col) or BASTA selected T2 pro35S:amiRNA-SCAP1 (amiRNA-SCAP1) lines. A transgenic line transformed with empty vector (vector) was used as a further control to account for BASTA treatment. Lines tested in this experiments are labelled in (B) with a filled arrowhead. Line \#2, white arrowhead in (B), was not included in this particular experiment. (D) Number of Guard cells (GC), pavement cells (PC) and stomatal index in wild type (Col) or BASTA selected T2 pro35S:SCAP1-YFP (35S:SCAP1) lines. In C and $D\left({ }^{* *}\right),\left({ }^{(X)}\right.$ ) and $\left({ }^{\circ}\right)=P<0.01$ (two tails T Student test) for comparisons between the wild type and the mutant alleles for GC, PC cell density or stomatal index, respectively. ns $=$ not significant. Values of $P C$ in (C) are all not significantly different compared with the wild type or vector. Error bars = Standard Error. (JPG $382 \mathrm{~kb}$ )

Additional file 3: SCAP1 regulates AtMYB60 expression. (A) AtMYB60 accumulation determined by quantitative PCR in manually dissected first two-leaf primordia of wild type (WT) scap1-2 and pro35S:amiRNA-SCAP1 (amiRNA2-SCAP1) seedlings at different time points. Values represent the mean of three biological replicates (30 leaves / replica). (B) SCAP1 transcript accumulations determined by RT-PCR in pro35S:SCAP1-GR T1 lines. Total RNA was isolated from 2-week-old seedlings and PCR was conducted for 30 cycles. Actin was used as a positive control and amplified for 25 cycles. (C) Transcript accumulation of AtMYB60 determined by quantitative PCR in DEX (or mock) treated wild type (Col) and pro35S:SCAP1-GR (35S:SCAP1-GR) transgenic plants at different time points. Values represent the mean of two biological replicates. In all quantitative PCR ACTIN (ACT2) was used for normalization. In $\mathrm{A}$ and $\mathrm{C},{ }^{* *}=\mathrm{P}<0.01$ and ${ }^{*}=\mathrm{P}<0.05$ and two tails $\mathrm{T}$ Student test. Error bars $=$ standard deviation. (D) Morphological alterations of stomata in cotyledons of GUS stained 4-weeks old single proAtMYB60:GUS WT (Col) or double proAtMYB60:GUS pro35S:SCAP1-GR (35S:SCAP1-GR) hemizygous plants. Bar $=20 \mu \mathrm{m}$. (E) Close up of GCs surrounded by clusters of meristemoids with altered spacing in cotyledons of DEX treated double proAtMYB60:GUS pro35S:SCAP1-GR (35S:SCAP1-GR) hemizygous plants (this plant was not subject to GUS staining) $\mathrm{Bar}=10 \mu \mathrm{m}$. (JPG $370 \mathrm{~kb}$ )

Additional file 4: Role of SCAP1 on stomatal genes transcript accumulations. (A) Transcript accumulation of stomatal markers $S P C H$, EPF2, MUTE and FAMA genes determined by quantitative PCR in manually dissected first two leaf primordia of 7 days-old wild type (Ler) and scap 1-2 plants. Values represent the mean of three biological replicates (30 leaves/ replica). (B-E) Transcript accumulation of stomatal markers EPF2, SPCH, MUTE and FAMA genes determined by quantitative PCR in 10 days-old DEX (or mock) treated wild type (Col) and (F-I) pro35S:SCAP1-GR (35S:SCAP1-GR) transgenic plants at different time point after treatment. Values represent the mean of two biological replicates. In all quantitative PCR ACTIN (ACT2) was used for normalization. Error bars $=$ standard deviation. ${ }^{*}=P<0.01$ and ${ }^{*}=\mathrm{P}<0.05$ and two tails T Student test. Values in (A) are all not significantly different compared with the wild type. (JPG $348 \mathrm{~kb}$ )

Additional file 5: Primers used in this study. (PDF $1736 \mathrm{~kb}$ )

\section{Abbreviations}

DEX, Dexamethasone; DOF, DNA Binding With One Finger; GC, Guard cell; GMC, guard mother cell; GR, Glucocorticoid Receptor; MMC, meristemoid mother cell; PC, Pavement cell; PDC, protodermal cell; SLGC, stomatal lineage ground cell; TF, Transcription Factor.

\section{Acknowledgements}

We would like to thank Prof. Enrico Coen for suggestions, the Nottingham Arabidopsis and the Cold Spring Harbour stock centres for providing T-DNA and Transposon tagged lines, Dr. Matsui (RIKEN Plant Functional Genomic) for the gift of the pro35S:SCAP1-GR construct. All the authors have no conflict of interest to declare.

\section{Funding}

GC was supported by a PhD fellowship from the University of Milan and, in part, by an Erasmus Student Placement Scheme. This work was supported by the Fondazione Umberto Veronesi, Milan (project AGRISOST) and in part by a MIUR PRIN project (2010-2011 prot. 2010HEBBB8_006) (to MG and CT).

\section{Availability of data and materials}

All supporting data are included as additional files. Transgenic lines, mutants and constructs described in this works are available upon request.

\section{Authors' contributions}

GC carried out the construction of transgenic plants, plant transformation and expression studies. GC and SF performed confocal studies. CT, MG, and LC conceived the study, participated in its design and coordination. GC, CT, $M G$ and LC wrote the manuscript. All authors read and approved the final manuscript.

\section{Competing interests}

The authors declare that they have no competing interests.

\section{Consent for publication}

Not applicable.

\section{Ethics approval and consent to participate}

Not applicable.

\section{Author details}

'Dipartimento di Bioscienze, Università degli studi di Milano, Via Celoria 26, 20133 Milan, Italy. ${ }^{2}$ Department of Cell and Developmental Biology, John Innes Centre, Norwich NR4 7UH, UK.

\section{Received: 15 March 2016 Accepted: 8 July 2016}

Published online: 02 August 2016

\section{References}

1. Dong J, MacAlister CA, Bergmann DC. BASL controls asymmetric cell division in Arabidopsis. Cell. 2009;137:1320-30.

2. Pillitteri LJ, Dong J. Stomatal development in Arabidopsis. Arabidopsis Book. 2013;11:e0162.

3. Geisler M, Nadeau J, Sack FD. Oriented asymmetric divisions that generate the stomatal spacing pattern in arabidopsis are disrupted by the too many mouths mutation. Plant Cell. 2000;12:2075-86.

4. Shpak ED, McAbee JM, Pillitteri LJ, Torii KU. Stomatal patterning and differentiation by synergistic interactions of receptor kinases. Science. 2005:309:290-3.

5. Hara K, Kajita R, Torii KU, Bergmann DC, Kakimoto T. The secretory peptide gene EPF1 enforces the stomatal one-cell-spacing rule. Genes Dev. 2007;21:1720-5.

6. Hara K, Yokoo T, Kajita R, Onishi T, Yahata S, Peterson KM, Torii KU, Kakimoto T. Epidermal cell density is autoregulated via a secretory peptide, EPIDERMAL PATTERNING FACTOR 2 in Arabidopsis leaves. Plant Cell Physiol. 2009:50:1019-31.

7. Hunt L, Gray JE. The signaling peptide EPF2 controls asymmetric cell divisions during stomatal development. Curr Biol. 2009;19:864-9.

8. Ohashi-Ito K, Bergmann DC. Arabidopsis FAMA controls the final proliferation/differentiation switch during stomatal development. Plant Cell. 2006:18:2493-505

9. MacAlister CA, Ohashi-Ito K, Bergmann DC. Transcription factor control of asymmetric cell divisions that establish the stomatal lineage. Nature. 2007; 445:537-40.

10. Pillitteri LJ, Sloan DB, Bogenschutz NL, Torii KU. Termination of asymmetric cell division and differentiation of stomata. Nature. 2007:445:501-5.

11. Kanaoka MM, Pillitteri LJ, Fujii H, Yoshida Y, Bogenschutz NL, Takabayashi J, Zhu J-K, Torii KU. SCREAM/ICE1 and SCREAM2 specify three cell-state transitional steps leading to arabidopsis stomatal differentiation. Plant Cell. 2008:20:1775-85.

12. Robinson S, Barbier de Reuille P, Chan J, Bergmann D, Prusinkiewicz P, Coen E. Generation of spatial patterns through cell polarity switching. Science. 2011;333:1436-40. 
13. Hunt L, Bailey KJ, Gray JE. The signalling peptide EPFL9 is a positive regulator of stomatal development. New Phytol. 2010;186:609-14.

14. Lee JS, Kuroha T, Hnilova M, Khatayevich D, Kanaoka MM, McAbee JM, Sarikaya M, Tamerler C, Torii KU. Direct interaction of ligand-receptor pairs specifying stomatal patterning. Genes Dev. 2012;26:126-36.

15. Lampard GR, MacAlister CA, Bergmann DC. Arabidopsis stomatal initiation is controlled by MAPK-mediated regulation of the bHLH SPEECHLESS. Science. 2008;322:1113-6.

16. Lampard GR, Lukowitz W, Ellis BE, Bergmann DC. Novel and expanded roles for MAPK signaling in Arabidopsis stomatal cell fate revealed by cell type-specific manipulations. Plant Cell. 2009;21:3506-17.

17. Bergmann DC, Lukowitz W, Somerville CR. Stomatal development and pattern controlled by a MAPKK kinase. Science. 2004;304:1494-7.

18. Jewaria PK, Hara T, Tanaka H, KONDO T, Betsuyaku S, Sawa S, Sakagami Y, Aimoto S, KAKIMOTO T. Differential effects of the peptides Stomagen, EPF1 and EPF2 on activation of MAP kinase MPK6 and the SPCH protein level. Plant Cell Physiol. 2013:54:1253-62.

19. Gudesblat GE, Schneider-Pizoń J, Betti C, Mayerhofer J, Vanhoutte I, van Dongen W, Boeren S, Zhiponova M, de Vries S, Jonak C, Russinova E. SPEECHLESS integrates brassinosteroid and stomata signalling pathways. Nature Cell Biol. 2012;14:548-54.

20. Engineer CB, Ghassemian M, Anderson JC, Peck SC, Hu H, Schroeder II. Carbonic anhydrases, EPF2 and a novel protease mediate CO2 control of stomatal development. Nature. 2014;513:246-50.

21. Lau OS, Davies KA, Chang J, Adrian J, Rowe MH, Ballenger CE, Bergmann DC. Direct roles of SPEECHLESS in the specification of stomatal self-renewing cells. Science. 2014;345:1605-9.

22. Wang H, Ngwenyama N, Liu Y, Walker JC, Zhang S. Stomatal development and patterning are regulated by environmentally responsive mitogenactivated protein kinases in Arabidopsis. Plant Cell. 2007;19:63-73.

23. Riechmann JL, Heard J, Martin G, Reuber L, Keddie J, Adam L, Pineda O, Ratcliffe OJ, Samaha RR, Creelman R. Arabidopsis transcription factors: genome-wide comparative analysis among eukaryotes. Science. 2000;290:2105-10.

24. Yanagisawa S. The Dof family of plant transcription factors. Trends Plant Sci. 2002;7:555-60

25. Skirycz A, Radziejwoski A, Busch W, Hannah MA, Czeszejko J, Kwaśniewski M, Zanor M-I, Lohmann JU, De Veylder L, Witt I, Mueller-Roeber B. The DOF transcription factor OBP1 is involved in cell cycle regulation in Arabidopsis thaliana. Plant J. 2008:56:779-92.

26. Galbiati M, Simoni L, Pavesi G, Cominelli E, Francia P, Vavasseur A, Nelson T, Bevan M, Tonelli C. Gene trap lines identify Arabidopsis genes expressed in stomatal guard cells. Plant J. 2008;53:750-62.

27. Cominelli E, Galbiati M, Albertini A, Fornara F, Conti L, Coupland G, Tonelli C. DOF-binding sites additively contribute to guard cell-specificity of AtMYB60 promoter. BMC Plant Biol. 2011;11:162.

28. Negi J, Moriwaki K, Konishi M, Yokoyama R, Nakano T, Kusumi K, Hashimoto-Sugimoto M, Schroeder Jl, Nishitani K, Yanagisawa S, Iba K. A Dof Transcription Factor, SCAP1, Is Essential for the Development of Functional Stomata in Arabidopsis. Curr Biol. 2013;23:479-84.

29. Cominelli E, Galbiati M, Vavasseur A, Conti L, Sala T, Vuylsteke M, Leonhardt N, Dellaporta SL, Tonelli C. A guard-cell-specific MYB transcription factor regulates stomatal movements and plant drought tolerance. Curr Biol. 2005;15:1196-200.

30. Aoyama T, Chua NH. A glucocorticoid-mediated transcriptional induction system in transgenic plants. Plant J. 1997:11:605-12.

31. Nadeau JA, Sack FD. Control of stomatal distribution on the Arabidopsis leaf surface. Science. 2002;296:1697-700.

32. Kondo T, Kajita R, Miyazaki A, Hokoyama M, Nakamura-Miura T, Mizuno S, Masuda Y, Irie K, Tanaka Y, Takada S, KAKIMOTO T, Sakagami Y. Stomatal density is controlled by a mesophyll-derived signaling molecule. Plant Cell Physiol. 2010;51:1-8

33. Sugano SS, Shimada T, Imai Y, Okawa K, Tamai A, Mori M, Hara-Nishimura I. Stomagen positively regulates stomatal density in Arabidopsis. Nature. 2010;463:241-4

34. Abrash EB, Bergmann DC. Regional specification of stomatal production by the putative ligand CHALLAH. Development. 2010;137:447-55.

35. Lee JS, Hnilova M, Maes M, Lin Y-CL, Putarjunan A, Han S-K, Avila J, Torii KU. Competitive binding of antagonistic peptides fine-tunes stomatal patterning. Nature. 2015;522:439-43.

36. Lake JA, Quick WP, Beerling DJ, Woodward FI. Plant development: signals from mature to new leaves. Nature. 2001;411:154-154.
37. Lake JA, Woodward Fl, Quick WP. Long-distance CO2 signalling in plants. J Exp Bot. 2002:53:183-93.

38. Coupe SA, Palmer BG, Lake JA, Overy SA, Oxborough K, Woodward Fl, Gray JE, Quick WP. Systemic signalling of environmental cues in Arabidopsis leaves. J Exp Bot. 2006:57:329-41.

39. Davies KA, Bergmann DC. Functional specialization of stomatal bHLHs through modification of DNA-binding and phosphoregulation potential. Proc Natl Acad Sci U S A. 2014;111(43):15585-90.

40. Clough SJ, Bent AF. Floral dip: a simplified method for Agrobacteriummediated transformation of Arabidopsis thaliana. Plant J. 1998;16:735-43.

41. Karimi M, Inzé D, Depicker A. GATEWAYTM vectors for Agrobacteriummediated plant transformation. Trends Plant Sci. 2002;7:193-5.

42. Ossowski S, Schwab R, Weigel D. Gene silencing in plants using artificial microRNAs and other small RNAs. Plant J. 2008;53:674-90.

43. Earley KW, Haag JR, Pontes O, Opper K, Juehne T, Song K, Pikaard CS. Gateway-compatible vectors for plant functional genomics and proteomics. Plant J. 2006:45:616-29.

44. Livak KJ, Schmittgen TD. Analysis of relative gene expression data using real-time quantitative PCR and the 2(-Delta Delta C(T)) Method. Methods. 2001;25:402-8.

\section{Submit your next manuscript to BioMed Central and we will help you at every step:}

- We accept pre-submission inquiries

- Our selector tool helps you to find the most relevant journal

- We provide round the clock customer support

- Convenient online submission

- Thorough peer review

- Inclusion in PubMed and all major indexing services

- Maximum visibility for your research

Submit your manuscript at www.biomedcentral.com/submit
Biomed Central 